Cahiers du MONDE RUSSE

\section{Cahiers du monde russe}

Russie - Empire russe - Union soviétique et États indépendants

$53 / 2-3 \mid 2012$

L'invention de la Sainte Russie

\title{
La construction de la sainte Russie
}

Les pèlerinages des gouverneurs moscovites au XVII ${ }^{\mathrm{e}}$ siècle

The construction of Holy Russia: Muscovite governors' pilgrimages in the seventeenth century

\section{Aleksandr Lavrov}

\section{(2) OpenEdition}

Journals

Édition électronique

URL : http://journals.openedition.org/monderusse/9388

DOI : $10.4000 /$ monderusse.9388

ISSN : $1777-5388$

Éditeur

Éditions de l'EHESS

Édition imprimée

Date de publication : 15 septembre 2012

Pagination : 375-384

ISSN : $1252-6576$

Référence électronique

Aleksandr Lavrov, "La construction de la sainte Russie », Cahiers du monde russe [En ligne], 53/2-3 | 2012, mis en ligne le 01 juillet 2015, Consulté le 03 mai 2019. URL : http://

journals.openedition.org/monderusse/9388; DOI : 10.4000/monderusse.9388

Ce document a été généré automatiquement le 3 mai 2019.

2012 


\title{
La construction de la sainte Russie
}

\author{
Les pèlerinages des gouverneurs moscovites au XVII ${ }^{\mathrm{e}}$ siècle \\ The construction of Holy Russia: Muscovite governors' pilgrimages in the \\ seventeenth century
}

Aleksandr Lavrov

1 «La notion de spiritualité est particulièrement exemplifiée dans le cas des personnages ayant un statut religieux ou théologique élevé. Mais avons-nous ici un exemple de la spiritualité des mousquetaires (strel'cy), des apprentis de l'Apothèque ou, surtout, de leurs femmes ? ", se demandait Frank Kämpfer dans son article consacré à un trouble religieux provoqué par la suppression des icônes privées dans une église moscovite en $1657^{1}$. Il semble que la recherche récente essaye vraiment de répondre à cette question, qui semblait à l'époque presque rhétorique, en trouvant de nouveaux exemples de la spiritualité (ou de la religiosité en général) des représentants des groupes non-privilégiés. C'est le cas de l'article de Sergej V. Rimskij, consacré aux pèlerinages des Cosaques du Don. Cet historien attire l'attention sur les chartes de passage (proezžie gramoty) qui furent délivrées aux Cosaques pour assurer leur pèlerinage vers le sanctuaire choisi. Ayant étudié cinquante-cinq pèlerinages, effectués entre 1640 et 1661, il montre une claire hiérarchie de l'adoration des saints chez les Cosaques. Ce sont les saints Zosima et Savvatij de Solovki (24), ainsi que les saints protecteurs de Moscou (20) qui attiraient surtout les cosaques-pèlerins. À la troisième place se trouvent les icônes et les monastères consacrés au culte de saint Nicolas de $\mathrm{Myra}^{2}$. Il est remarquable que l'historien n'avance pas tout de suite une explication complète de tous les phénomènes qu'il décrit, mais avoue que, pour le moment, il n'a pas une «réponse claire " sur les causes de la popularité des saints de Solovki³.

2 En travaillant dans les Archives des actes anciens à Moscou, j'ai trouvé plusieurs actes manuscrits portant sur des pèlerinages dont le témoignage pourrait être comparé à celui des documents étudiés par S.V. Rimskij. Il s'agit de neuf suppliques de gouverneurs de villes (voevody) qui voulaient partir en pèlerinage ${ }^{4}$.

Dans leurs suppliques les gouverneurs demandaient la permission de quitter la bourgade qui leur était confiée (si on transposait à notre époque, on pourrait parler de « demande 
d'autorisation d'absence»). Pour argumenter leur demande, ils devaient proposer la candidature d'un remplaçant - généralement, un noble retraité - et limiter leur absence à un ou deux mois. Normalement, la permission était accordée. Voici un exemple d'une supplique ayant abouti :

Aux tsars et grands princes (titre) Ivan Alekseevič et Petr Alekseevič et à la grande souveraine, pieuse tsarevna et grande princesse Sofija Alekseevna... votre esclave, Eremko Paškov, se prosterne. Selon votre édit souverain je suis nommé gouverneur à Epifan', et moi, votre esclave, j'ai fait le vœu de prier au monastère de SaintNicolas à Radunicy dans le district de Rjazan'. Je n'ose pas aller d'Epifan' à ce monastère sans votre ordre souverain. Miséricordieux tsars.... et pieuse grande princesse...., donnez-moi la permission, ordonnez de me laisser dans ce monastère pour la prière pour le délai de votre choix. Grands souverains, ayez pitié, donnez votre permission. ${ }^{5}$

4 Vers la fin des années 1690 l'argumentation devient plus élaborée, ce qui laisse supposer que pendant cette période l'obtention de la permission d'aller en pèlerinage n'était plus automatique. Les auteurs n'évoquent pas seulement un vœu pieux, mais aussi l'état désastreux de leur santé et l'absence d'obligations administratives urgentes.

Je suis nommé gouverneur de Starica selon ton ordre souverain et, par la volonté du grand Dieu, je suis malade depuis plusieurs jours [...] et je n'ai maintenant à Starica [...] aucune affaire avec un demandeur ou un défendeur, et les affaires criminelles me sont retirées suivant ton ordre souverain,

écrivait Jakov Goleniščev-Kutuzov. Cette supplique fut acceptée, mais, contrairement à l'expérience précédente, Goleniščev-Kutuzov n'obtint pas plus d'un mois pour son pèlerinage (contre les deux qui étaient traditionnellement accordés au XVII ${ }^{\mathrm{e}}$ siècle) ${ }^{6}$. Je ne connais aucun cas de refus - évidemment, tous les gouvernements moscovites du XVII ${ }^{\mathrm{e}}$ siècle, de celui de Mihail Fedorovič jusqu'à celui de Pierre le Grand, considéraient les pèlerinages comme légitimes. Les sources ne donnent aucune possibilité de savoir si les gouverneurs partaient en pèlerinage seuls ou accompagnés de leurs épouses et de leurs proches.

5 J'ai trouvé trois autres suppliques avec une demande d'autorisation à aller à un pèlerinage, mais leurs auteurs ne sont pas gouverneurs, ce qui explique que je n'introduise pas leurs données dans la statistique générale. Il s'agit tout d'abord de la supplique d'un Ivan Detkovskij, dont je suppose qu'il était un noble de Moscou (moskovskij dvorjanin). Cette appartenance probable aux grades de Moscou rapproche Detkovskij des gouverneurs? ${ }^{7}$. Mais il n'envoya pas sa supplique de son lieu de service, plutôt probablement de Moscou. Son cas était un peu particulier. «Et moi, ton esclave, je fus exilé en Sibérie à cause de ta disgrâce souveraine et, me trouvant là-bas, j’ai fait le vœu (d'aller) aux thaumaturges de Solovki ", écrit-il dans sa supplique ${ }^{8}$. Je propose la lecture suivante, strictement hypothétique : se trouvant en exil, Detkovskij fit le vœu que, en cas de pardon du Tsar et de retour dans la partie européenne de la Moscovie, il irait en pèlerinage à Solovki. La permission lui fut accordée.

6 Les deux autres suppliques émanent d'esclaves (xolopy) : normalement, ceux-ci laissaient peu de traces dans la documentation pourtant abondante de la Moscovie. Ici, l'objectif de la demande était un peu différent : les auteurs avaient besoin d'une lettre confirmant le pieux objectif de leur déplacement, pour que les gouverneurs des villes les laissent passer et ne les traitent pas comme des esclaves en fuite. Ces deux suppliques sont signées par trois esclaves et portent toutes deux sur le pèlerinage au monastère de Solovki ${ }^{9}$. 
7 La systématisation des données des neuf suppliques de gouverneurs, auxquelles on ajoute, à titre de comparaison, une supplique d'un exilé et trois suppliques d'esclaves, peut être effectuée de la manière suivante:

\begin{tabular}{|c|c|c|c|c|c|}
\hline Année & $\begin{array}{l}\text { Nom, prénom, } \\
\text { patronyme }\end{array}$ & Rang & $\begin{array}{c}\text { Lieu de } \\
\text { départ }\end{array}$ & $\begin{array}{c}\text { Lieu de } \\
\text { pelerinage }\end{array}$ & $\begin{array}{l}\text { Date de dépôt } \\
\text { de la demande }\end{array}$ \\
\hline 1688 & $\begin{array}{l}\text { Paškov Eremej } \\
\text { Davydovič }\end{array}$ & panetier & Epifan' & $\begin{array}{l}\text { Monastère } \\
\text { de Radunicy }\end{array}$ & $\begin{array}{l}\text { avant } \\
\text { le } 19 \text { mai }\end{array}$ \\
\hline 1689 & \begin{tabular}{|l|} 
Zolotarev Ivan \\
Vasil'evič \\
\end{tabular} & & Kaluga & $\begin{array}{l}\text { Gostuni, Nikola } \\
\text { Belevskij }\end{array}$ & $\begin{array}{l}\text { Avant } \\
\text { le } 11 \text { octobre }\end{array}$ \\
\hline 1698 & $\begin{array}{l}\text { Lagovčin Vasilij } \\
\text { Ivanov }\end{array}$ & panetier & Kromy & Moscou & $\begin{array}{l}\text { avant } \\
\text { le } 18 \text { novembre }\end{array}$ \\
\hline 1694 & L'vov Ivan Andreev & panetier & Vorotynsk & Moscou & $\begin{array}{l}\text { avant } \\
\text { le } 21 \text { iuillet }\end{array}$ \\
\hline 1696 & $\begin{array}{l}\text { Ofrosimov Semen } \\
\text { Mihajlov }\end{array}$ & panetier & Bolhov & $\begin{array}{l}\text { Nikolaj na } \\
\text { Epifani i Pjatnica } \\
\text { v Veneve }\end{array}$ & $\begin{array}{l}\text { avant } \\
\text { le } 26 \text { juillet }\end{array}$ \\
\hline 1696 & Loginov Semen & & Karačev & Gostuni, Nikola & $\begin{array}{l}\text { avant } \\
\text { le } 12 \text { juin }\end{array}$ \\
\hline 1696 & \begin{tabular}{|l} 
Tarbeev Dmitrij \\
Dmitriev
\end{tabular} & & Pošexon'e & Kirillov & $\begin{array}{l}\text { avant } \\
\text { le } 11 \text { mars }\end{array}$ \\
\hline 1696 & $\begin{array}{l}\text { Goleniščev-Kutuzov } \\
\text { Jakov Ivanov }\end{array}$ & & Starica & $\begin{array}{l}\text { Toropec, } \\
\text { Korsunskaja }\end{array}$ & $\begin{array}{l}\text { avant } \\
\text { le } 5 \text { octobre }\end{array}$ \\
\hline 1696 & $\begin{array}{l}\text { Goleniščev-Kutuzov } \\
\text { Jakov Ivanov }\end{array}$ & & Starica & $\begin{array}{l}\text { Toropec, } \\
\text { Korsunskaja }\end{array}$ & $\begin{array}{l}\text { octobre- } \\
\text { novembre }\end{array}$ \\
\hline 1644 & Detkovskij Ivan & exilé & Sibérie & Solovki & $\begin{array}{l}\text { avant } \\
\text { le } 30 \text { mai }\end{array}$ \\
\hline $1613-1645$ & Osipov Sergej & $\begin{array}{l}\text { esclave du secr. } \\
\text { Gr. L'vov }\end{array}$ & Moscou & Solovki & avril \\
\hline $1613-1645$ & Danilov Ivan & \begin{tabular}{|l} 
esclave d'And. \\
Vas.Hilkov \\
\end{tabular} & Moscou & Solovki & avril \\
\hline 1645 & Pavlov Karp & $\begin{array}{l}\text { esclave du secr. } \\
\text { Gr. Larionov }\end{array}$ & Moscou & Solovki & mars \\
\hline
\end{tabular}

Malgré le nombre plus que modeste des suppliques, plusieurs questions peuvent être posées. Comment s'effectuait le pèlerinage d'un noble moscovite, quel était son cadre spatio-temporel? Quels étaient les cultes préférés des pèlerins? En partant de ce choix des cultes, est-il possible de reconstruire une religiosité noble à part ou, au contraire, ces cultes s'inscrivent-ils plus ou moins dans le cadre de l'« orthodoxie populaire »?

En essayant de répondre à la première question, sur le cadre spatio-temporel des pèlerinages ${ }^{10}$, il faut tenir compte $d u$ fait que les gouverneurs moscovites étaient les représentants du centre envoyés à la périphérie. Ils pouvaient mettre à profit leurs séjours dans les districts éloignés pour visiter tel ou tel sanctuaire, voisin de leur lieu d'affectation. Dans ce cas, on peut parler de la construction mentale des espaces périphériques de la Moscovie, qui furent repensés non seulement comme des «marges " ou des «frontières ", mais aussi comme des «lieux de mémoire », possédant un certain statut sacral.

La majorité des demandes des gouverneurs était liée à la visite d'un lieu saint, qui ne se trouvait pas directement dans le district qui leur était confié, mais était plus proche et plus accessible depuis leur lieu d'affectation qu'à partir de Moscou. Par exemple, Jakov Goleniščev-Kutuzov, dont la supplique a déjà été citée, gouverneur de Starica (au nordouest de Moscou), voulait partir à Toropec (encore plus vers le Nord-Ouest). Dmitrij Tarbeev, envoyé à Pošehon'e (au nord de Moscou), partait à Kirillov-Belozerskij, monastère (vers le Nord). Ivan Zolotarev, envoyé à Kaluga (au sud-ouest de Moscou), partait à Belev (encore plus vers le Sud-Ouest). Ces deux pèlerinages, pendant lesquels les pèlerins devaient parcourir 180, 200 ou 250 kilomètres, peuvent être représentés graphiquement comme des vecteurs dirigés du centre de la Moscovie vers sa périphérie. 
11 Le pèlerinage de Paškov d'Epifan' au monastère de Radunickij (150 kilomètres) était aussi un voyage de la province vers la province, mais ici la direction était un peu différente, on peut représenter ce voyage comme une tangente à un cercle dont le centre est Moscou. Le voyage de Semen Loginov constitue une exception: pour venir de Karačev à Belev, il devait parcourir cent vingt kilomètres dans la direction de Moscou (soit un vecteur dirigé de la périphérie vers le centre). Ainsi, tous les gouverneurs qui ont choisi leurs lieux de pèlerinage en province ont aussi choisi des lieux saints relativement proches de leur lieu de service.

Deux pèlerinages ne s'inscrivent pas dans cette logique : ceux de Vasilij Lagovčin et d'Ivan L'vov qui voulaient vénérer les «thaumaturges de Moscou ». C'est un clair retour de la périphérie au centre. Nous n'avons aucun élément pour affirmer que les deux pèlerins nobles cherchaient tout simplement un prétexte pour visiter la capitale. Il est incontestable qu'un bref séjour à Moscou leur permettait de régler leurs affaires dans les secrétariats et de voir leurs proches et leurs amis, mais présentait aussi une offre religieuse et culturelle assez large. Par contre, les petites villes provinciales choisies par leurs collègues pour leurs pèlerinages ne pouvaient rien offrir d'autre que le sanctuaire. Dans ce dernier cas, c'est-à-dire, dans le cas majoritaire, le motif religieux devait être dominant.

Dans presque tous les cas, quand la date de la commémoration du saint et celle du pèlerinage prévu sont connues, on peut mettre en évidence une relation. Dans le cas de sainte Parascève, la seule date de commémoration est le 28 octobre, tandis que la supplique de Semen Ofrosimov fut déposée avant le 26 juillet. Dans le cas de Nicolas de Myra, on peut choisir entre le jour de sa mort (le 9 mai), auquel renvoie la supplique d'Eremej Paškov (déposée avant le 19 mai), et le 6 décembre, le jour du transfert de ses reliques à Bari (voir la supplique d'Ivan Zolotarev, déposée avant le 11 octobre). Il est possible que Jakov Goleniščev-Kutuzov soit parmi les retardataires: il ne déposa sa supplique que le 5 octobre, tandis que la fête de l'icône Korsunskaja était le 9 octobre. Cependant, on ne sait pas si à Toropec on respectait cette dernière date pour célébrer cette icône miraculeuse. Le cas de Dmitrij Tarbeev est moins évident: il déposa sa supplique avec la demande de permission d'aller au monastère de Saint-Kirill avant le 11 mars, tandis que le jour de la commémoration de saint Kirill de Beloozero est le 9 juin. Cependant, même cet intervalle peut-être expliqué. Pour sa correspondance avec Moscou, Tarbeev utilisait les voies de communication praticables en hiver (zimnij put'), et il est possible qu'il ait reçu sa permission avant la saison des mauvaises routes (rasputica). En tout cas, je pense qu'il a dû être bloqué les deux mois suivants et qu'en agissant ainsi, il a pu rejoindre le monastère pour la date solennelle ${ }^{11}$.

En essayant de répondre à la deuxième question et de caractériser les cultes choisis, nous nous heurtons à plusieurs surprises. Tout d'abord, les cultes les plus renommés ne semblaient pas attirer les pèlerins nobles. L'exemple le plus frappant est celui de la Trinité-Saint-Serge qui est totalement absente des vœux des gouverneurs, ce qui les rapproche des Cosaques du Don. L'étatisation du culte de saint Serge, évident au XVII siècle, l'a-t-elle rendu moins attirant pour les pèlerins, privilégiés et non privilégiés ? De plus, le pèlerinage à Solovki, qui était le plus pratiqué chez les Cosaques, est aussi absent chez les gouverneurs. Si on se souvient que les trois esclaves et un exilé ont eux aussi choisi Solovki, on ne peut pas éviter l'hypothèse selon laquelle ce pèlerinage revêt déjà au $\mathrm{xvII}^{\mathrm{e}}$ siècle un caractère protonational. De plus, aucun gouverneur ne choisit le culte d'un saint prince ${ }^{12}$, bien qu'un tel intérêt semble typique d'une religiosité noble. 

les nouveaux, en formation. Quelques pèlerinages de gouverneurs se réfèrent aux cultes qui se développaient autour des anciens sanctuaires, qui avaient acquis une attractivité interrégionale récente et qui seront par la suite presque abandonnés. Les cultes de l'icône de la Mère de Dieu Toropeckaja-Korsunskaja et le culte de saint Nicolas Gostunskij relèvent de ce groupe. Il est particulièrement intéressant d'observer que les sites de ces deux cultes n'étaient pas monastiques.

Comme nous le savons déjà, la demande du pèlerinage à l'icône Korsunskaja à Toropec se trouve dans la supplique de Jakov Goleniščev-Kutuzov. Il s'agit de l'Hodiguitria

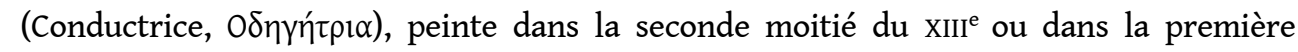
moitié du XIv ${ }^{\mathrm{e}}$ siècle, qui se trouvait dans la collégiale de Toropec. Selon la légende, cette icône fut apportée au pays de Novgorod par la princesse de Polock, la fiancée du prince Aleksandr Nevskij. Le Dit (Skazanie) sur cette icône n'apparaît qu'à la fin du XvII ${ }^{\mathrm{e}}$ siècle, au début $\mathrm{du} \mathrm{xvIII}^{\mathrm{e}}$ siècle. Ainsi, la supplique de Goleniščev-Kutuzov est-elle une des premières preuves de l'attractivité du culte de l'icône à dépasser le cadre local ${ }^{13}$.

Troisièmement, se pose la question épineuse de la relation entre la subculture religieuse de la noblesse moscovite, que nous essayons de reconstruire ici, et l'« orthodoxie populaire ». À notre avis, deux éléments sont ici pertinents : le culte de saint Nicolas et le rôle des images sculptées. L'icône de Saint-Nicolas de Gostuni attira deux pèlerins, Ivan Zolotarev et Semen Loginov. C'était une icône miraculeuse, trouvée à la fin du xvi siècle près du village de Gostuni, sur la rive droite de l'Oka, entre Lihvin et Belev. En 1506, l'icône fut transportée au Kremlin, où le grand prince Vasilij Vasil'evič édifia pour elle une église. Ivan Fedorov, le fondateur de l'imprimerie moscovite, était alors diacre de cette église. La copie de l'icône resta dans le village de Gostuni ${ }^{14}$. Évidemment, le culte fonctionnait de manière binaire, l'église du Kremlin, connue de tous les membres de la cour, suscitait l'intérêt pour Gostuni, le site initial du culte, bien qu'on ne pût y trouver que la copie, tandis que l'icône originale se trouvait au Kremlin. On peut dire que pendant les $\mathrm{XVIII}^{\mathrm{e}}$ et $\mathrm{XIX}^{\mathrm{e}}$ siècles ce pèlerinage perd de sa popularité.

Il n'est pas facile de dire s'il s'agit ici d'une icône peinte ou d'une image sculptée. On trouve dans les collections des musées des icônes, avec l'iconographie traditionnelle de saint Nicolas et de sa Vita (s žitiem), qui portent le titre de «Saint-Nicolas de Gostuni ». En même temps, nous avons au moins une statue de saint Nicolas, avec l'iconographie traditionnelle de Saint-Nicolas de Možajsk (c'est-à-dire, avec le glaive et avec le modèle de la ville), qui porte le titre de "Saint-Nicolas de Gostuni» (elle provient de l'église de Saint-Nicolas Gostunskij au Kremlin) ${ }^{15}$. On pourrait même avancer l'hypothèse de l'existence de deux icônes identiques, dont l'une se trouvait à Moscou et l'autre à Gostuni, et de deux sculptures identiques, dont l'une se trouvait à Moscou et l'autre à Gostuni. Le culte initial se développait-il autour d'une sculpture ou autour d'une icône ? La sculpture ou l'icône était-elle au centre du culte au moment du pèlerinage étudié ? Pour le moment, il est difficile de donner une réponse définitive.

On peut avancer l'hypothèse selon laquelle l'icône de Saint-Nicolas à Epifan', mentionnée dans la supplique de Semen Ofrosimov, serait tridimensionnelle. Les témoignages qui se réfèrent à la première moitié $\mathrm{du} \mathrm{xx}^{\mathrm{e}}$ siècle décrivent cette icône (obraz) comme grande et «sculptée $(r e z n o j) »^{16}$. Les processions avec cette icône considérée comme miraculeuse attiraient les paysans. Bien sûr, tout cela n'est pas pertinent pour le XVII siècle, car le caractère du culte a pu changer au cours des XVIII et XIX ${ }^{e}$ siècles. 
20 Malheureusement, on ne peut dire grand-chose sur la seconde icône que Semen Ofrosimov voulait vénérer, l'icône de Parascève à Venev. Dans la description de la ville, faite après l'incursion des Tatares au XVII ${ }^{\mathrm{e}}$ siècle, on évoque «l'icône de la Parascève Pjatnica (Vendredi) dans un revêtement d'argent, avec le nimbe en filigrane et avec les ménisques ciselés suspendus ( $v$ serebrjanom oklade, $v$ skannom vence is catami basmennymi)» ${ }^{17}$. Mais l'apparition commune de ces deux saints, saint Nicolas et sainte Parascève, est très significative. Selon A.V. Pigin, la réunion des noms de saint Nicolas, sainte Barbara et sainte Parascève est caractéristique des textes qui se réfèrent à l'« orthodoxie populaire » 18 .

21 Il est cependant un cas où il s'agit indiscutablement d'un pèlerinage vers une image sculptée. C'est celui de la supplique du panetier Eremej Davydovič Paškov (1688), qui voulait se rendre à l'icône de saint Nicolas au monastère de Radovicy. Selon l'iconographie, le Saint-Nicolas de Radovicy était présenté avec le glaive dans une main et le modèle de la ville dans l'autre (comme Saint-Nicolas de Možajsk). Mais le Saint-Nicolas de Radovicy avait la mitre sur la tête, contrairement au Saint-Nicolas de Možajsk ${ }^{19}$.

Pour autant, il n'est pas simple de mettre un signe égal entre ces quatre pèlerinages et l'« orthodoxie populaire». Tandis que les historiens de l'art ont beaucoup contribué à l'étude de la sculpture russe médiévale, les jugements des historiens de la religion restent partagés ${ }^{20}$. Selon l'opinion la plus répandue, la sculpture est un témoignage de la christianisation tardive, une sorte de compromis entre l'orthodoxie et la tradition préchrétienne de la vénération des images sculptées. Il est légitime de se demander si cette explication est suffisante pour comprendre l'intérêt des nobles moscovites. Leur intérêt pour les icônes sculptées pourrait s'expliquer par l'influence de la piété catholique, surtout polonaise, qui acceptait volontiers les images sculptées; cet intérêt pourrait être interprété dans le cadre de la religiosité noble.

Le classement devient encore plus difficile dans le cas où le culte en question est bien documenté. Pour le culte de Saint-Nicolas de Radovicy, nous connaissons une liste de guérisons, observées entre 1619 et 1722, qui contient 266 noms. La majorité des personnes guéries appartient à la paysannerie locale. Nous pourrions dire qu'il s'agit d'un culte local, majoritairement paysan. Mais cela n'empêchait pas les aristocrates moscovites de participer à ce culte. Les nobles préférèrent le rôle de " donateurs ", laissant aux paysans le rôle de « témoins » des miraculés ${ }^{21}$. Nous trouvons parmi les donateurs du monastère la tsarine Natal'ja Kirilovna, le boyard Kirill Naryškin et la tsarine Evdokija Fedorovna (c'est-à-dire, la mère, le grand-père et la première femme de Pierre le Grand), mais aussi sa sœur consanguine Sof ja Alekseevna et ses parents les Miloslavskie ${ }^{22}$. L'intégration de ce culte dans la piété de la famille tsarienne et de l'aristocratie moscovite, et le fait que le culte devient connu à Moscou, présupposent sa répression pendant la « réforme de la piété » de Pierre le Grand. En 1722 l'icône sculptée de Radovicy fut confisquée et apportée au saint-synode ${ }^{23}$. C'est seulement en 1728 , dans le cadre d'une "contre-réforme " religieuse, que l'icône fut rendue à Radovicy, accompagnée par une procession solennelle 24 .

24 Cette petite recherche ne peut être considérée que comme une première approche du sujet: pour le moment, il est difficile de dire combien de telles pétitions se trouvent encore dans les archives, car les éditeurs de la Description des documents et de papiers, conservés aux archives moscovites du ministère de la justice semblent ne pas indiquer de façon systématique leur présence dans les rouleaux (stolbcy), ainsi, certaines pétitions ne furent retrouvées que par hasard. Malgré cela, on pourrait parler de trois résultats principaux. 
D'abord, les pèlerins du XVII ${ }^{e}$ siècle ne se rendaient pas aux mêmes sanctuaires que les pèlerins du XVIII ${ }^{\mathrm{e}}$ siècle étudiés par Christine Worobec ${ }^{25}$. Deux exceptions cependant, les pèlerinages vers les sanctuaires de Moscou et vers le monastère de Solovki, montrent clairement les cultes avec une dimension panrusse qui se formèrent au XVII ${ }^{\mathrm{e}}$ siècle et qui gardèrent leur importance jusqu'au $\mathrm{xx}^{\mathrm{e}}$ siècle. Par contre, plusieurs pèlerinages, qu'on peut repérer grâce aux suppliques, étaient liés à des cultes fragiles, qui pouvaient très vite perdre de leur importance pour les croyants.

De plus, nous trouvons des églises paroissiales parmi les sites de pèlerinage. Ce n'était possible qu'avant la réforme de Pierre le Grand, qui visa à concentrer les reliques et les icônes vénérées dans les cathédrales et les monastères.

Enfin, on pourrait parler avec prudence de nouveaux éléments dans les pèlerinages. C'est notamment le cas du pèlerinage de Semen Ofrosimov à Epifan', qui conduisait le pèlerin à la frontière du Champ des bécasses (Kulikovo pole). Même la légende de l'icône de SaintNicolas d'Epifan' raconte que cette icône fut trouvée par des guerriers russes revenant après la bataille au Champ des bécasses en $1380^{26}$. Ainsi, le pèlerinage vers Epifan' se transformait-il en un voyage vers l'un des principaux « lieux de mémoire » de l'histoire russe $^{27}$. Il ne s'agit pas pour moi ici de séculariser le pèlerinage de Semen ofrosimov en le rapprochant du tourisme cultuel moderne. D'ailleurs, ce pèlerinage s'inscrit parfaitement dans le calendrier liturgique, comme la majorité des pèlerinages étudiés. En revanche, il serait très intéressant d'entreprendre une micro-étude de cette famille noble pour savoir si la référence au Champ des bécasses était tout simplement protonationale, ou personnelle.

\section{NOTES}

1. Frank Kämpfer, «Verhöre über das Entfernen von Ikonen aus den Kirchen. Ein Vorgang aus dem Moskau des Jahres 1657», in Unser ganzes Leben... Festschrift Fairy von Lilienfeld, Göttingen, 1982, p. 295-302. Je suis reconnaissant à Christine Worobec, Elena Smiljanskaja et Nadežda Pivovarova pour leurs précieuses remarques qui m'ont permis de préciser certains points de cet article.

2. S.V. Rimskij, «Počitanie svjatyh donskimi kazakami [L'adoration des saints par les cosaques du Don] », Problema svjatyh i svjatosti v istorii Rossii. Materialy XX Meždunarodnogo seminara istoričeskih issledovanij «Ot Rima $k$ Tret'emu Rimu ». Problema svjatyh $i$ svjatosti $v$ kontekste istorii i prava [Des saints et de la sainteté dans l'histoire russe. Travaux du $\mathrm{XX}^{\mathrm{e}}$ séminaire des études historiques "De Rome à la Troisième Rome ", Des saints et de la sainteté dans le contexte de l'histoire et du droit], M., 2006, p. 217-231. Les chartes étudiées par $\mathrm{S}$. Rimskij furent publiées au début $\mathrm{du} \mathrm{xx}^{\mathrm{e}}$ siècle, mais n'ont pas retenu jusqu'à maintenant l'attention des historiens.

3. Ibid., p. 230. Sur le culte des saints Zosima et Savvatij de Solovki, voir l'édition monumentale des sources et l'étude de S.V. Mineeva, Rukopisnaja tradicija Žitija prep. 
Zosimy i Savvatija [La tradition manuscrite des Vitae des saints Zosima et Savvatij], t. 1-2, M. : Jazyki slavjanskih kul'tur, 2001.

4. RGADA (Rossijskij gosudarstvennyj arhiv drevnih aktov - Archives russes des actes anciens), f. 210 (Razrjadnyj prikaz), Stolbcy Novgorodskogo stola, $\mathrm{n}^{\circ} 239,1.4 \mathrm{~b} ; 1.20 ; \mathrm{n}$ ○331, 1. 96; Stolbcy Sevskogo stola, n 35, 1. 30, 118; n 63, 1. 237; n 66, 1. 334; n 74, 1. 102, 457.

5. «В.Г.Ц. И В.КН. ИОАННУ АЛЕКСЕЕВИЧУ, ПЕТРУ АЛЕКСЕЕВИЧУ И ВЕЛИКОЙ ГОСУДАРЫНЕ БЛАГОВЕРНОЙ ЦАРЕВНЕ И ВЕЛИКОЙ КНЯЖНЕ СОФИИ АЛЕКСЕЕВНЕ В. В.И М. И Б. Р. С. БЬЕТ ЧЕЛОМ ХОЛОП ВАШ ЕРЕМКО ПАШКОВ. ПО ВАШЕМУ ВЕЛИКИХ ГОСУДАРЕЙ УКАЗУ Я ХОЛОП ВАШ НА ЕПИФАНЕ ВОЕВОДОЮ, А ОБЕЩАНИЕ У МЕНЯ, ХОЛОПА ВАШЕГО, В РЕЗАНСКОМ УЕЗДЕ В МОНАСТЫРЬ К ЧУДОТВОРЦУ НИКОЛЕ В РАДУНИЦЫ ПОМОЛИТЦА, А БЕЗ ВАШЕГО ВЕЛИКИХ ГОСУДАРЕЙ УКАЗУ Я, ХОЛОП ВАШ, С ЕПИФАНИ В ТОТ МОНАСТЫРЬ ЕХАТЬ НЕ СМЕЮ. МИЛОСЕРДЫЕ В.Г.Ц. И В. КН. ИОАНН АЛЕКСЕЕВИЧЬ, ПЕТР АЛЕКСЕЕВИЧЬ И ВЕЛИКАЯ КНЯЖНА СОФИЯ АЛЕКСЕЕВНА В. В.И М. И Б. Р.С., ПОЖАЛУЙТЕ МЕНЯ, ХОЛОПА СВОЕГО, ВЕЛИТЕ, ГОСУДАРИ, МНЕ ХОЛОПУ СВОЕМУ В ТОТ МОНАСТЫРЬ ДЛЯ МОЛЕНИЯ ОТПУСТИТЬ НАСТОЛЬКО ВЫ, В.Г., УКАЖИТЕ. ВЕЛИКИЕ ГОСУДАРИ, СМИЛУЙТЕСЯ, ПОЖАЛУЙТЕ», RGADA, f. 210, Stolbcy Sevskogo stola, n 63, l. 237.

6. «ПО ТВОЕМУ ВЕЛИКОГО ГОСУДАРЯ УКАЗУ НЫНЕ Я, ХОЛОП ТВОЙ, В СТАРИЦЕ ВОЕВОДОЮ, И ВОЛЕЮ ВЕЛИКОГО БОГА В БОЛЕЗНИ ЛЕЖУ МНОГИЕ ЧИСЛА, И ОБЕЩАЛСЯ Я, ХОЛОП ТВОЙ, ИС СТАРИЦЫ ЕХАТЬ В ТОРОПЕЦ ПОМОЛИТЦА ОБРАЗУ ПРЕСВЯТЫЕ БОГОРОДИЦЫ, И НЫНЕ У МЕНЯ, ХОЛОПА ТВОЕГО, В СТАРИЦЕ ЗА ТВОИМ ВЕЛИКОГО ГОСУДАРЯ УКАЗОМ У ИСЦОВ С ОТВЕТЧИКАМИ НИКАКИХ ДЕЛ НЕТ, А ГУБНЫЕ ДЕЛА У МЕНЯ ХОЛОПА ТВОЕГО ОТНЯТЫ», RGADA, f. 210, Stolbcy Novgorodskogo stola, $\mathrm{n}^{\circ} 239,1.10-10 v e r$., Supplique de Jakov Ivanov Goleniščev-Kutuzov, avant 5 octobre 1696.

7. L'identification d'Ivan Detkovskij ne va pas de soi. Le noble de Moscou Ivan Detkovskij est évoqué dans le registre de la cour (bojarskaja kniga) 7148 (N 4). Dans le registre de la cour suivant est évoqué un certain Ivan Ivanovič Detkovskij, qui est aussi noble de Moscou. S'agit-il du même personnage? Dans ce cas, Detkovskij revint de son exil sibérien et son grade lui fut restitué. Ivan Ivanovič Detkovskij est-il le fils d'Ivan Detkovskijl'exilé ? Dans ce cas, on doit reconnaître que le destin de l'exilé reste inconnu. P. Ivanov, Alfavitnye ukazateli familij $i$ lic, upominaemyh $v$ bojarskih knigah [Listes alphabétiques des personnages mentionnés dans les registres de la cour], M., 1853, p. 125.

8. «СОСЛАН БЫЛ Я, ХОЛОП ТВОЙ, В СИБИРЬ ЗА ТВОЮ ГОСУДАРЕВУ ОПАЛУ И БУДУЧИ ТАМ ОБЕЩАЛСЯ СОЛОВЕЦКИМ чюДОТвоРЦАМ», RGADA, f. 210 (Razrjadnyj prikaz), Stolbcy Belgorodskogo stola, $\left.n^{\circ} 169,1.197\right)$.

9. RGADA, f. 210 (Razrjadnyj prikaz), Stolbcy Belgorodskogo stola, n 169, 1. 199; Stolbcy Belgorodskogo stola, $\mathrm{n}^{\circ} 283,1.274$.

10. Sur les pèlerinages en général voir: H.V. Poplavskaja, «Palomničestvo, strannopriimničestvo i počitanie svjatyn' (po materialam Rjazanskogo kraja) [Le pèlerinage, l'hospitalité et le culte de sanctuaires (selon les données de la région de Rjazan')]", in Pravoslavnaja žizn' russkih krest'jan XIX-XX vekov. Itogi ètnografičeskih issledovanij [La vie orthodoxe des paysans russes aux $\mathrm{xIX}^{\mathrm{e}}-\mathrm{Xx}^{\mathrm{e}}$ siècles. Les résultats des études ethnographiques], M., 2001, p. 251-299.

11. La supplique d'Ivan Detkovskij présente le cas le plus complexe. Elle fut reçue vers la fin mai. Pour venir de Moscou à Arhangel'sk ou à Kem', on devait voyager pendant l'hiver et l'été. Pour atteindre l'archipel de Solovki, il fallait se trouver à Arhangel'sk ou à Kem' 
pendant la période de navigation. Ainsi, le commencement de l'été présentait-il le meilleur moment pour ce pèlerinage. Il me semble que dans ce cas la date fut plutôt définie par les contraintes de la communication.

12. Sur les cultes des saints princes voir: Gail Lenhoff, Early Russian Hagiography: The Lives of Prince Fedor the Black (= Slavistische Veröffentlichungen Fachbereich Neuere Fremdsprachliche Philologien der Freien Universität Berlin, Bd: 82), Wiesbaden, 1997.

13. "Prečistomu obrazu Tvoemu poklonjaemsja..." Obrazy Bogomateri v proizvedenijah iz sobranija Russkogo muzeja ["Nous nous inclinons devant Ton image Très-pure... », Les images de la Mère de Dieu dans les collections du Musée Russe), comp. N. Pivovarova, I. Šalina, T. Vilinbahova, A. Mal'ceva, N. Solov'eva, I. Sosnovceva, N. Turcova, M. Fedoseeva, SPb., 1995, p. 181, N 105 ; O.E. Ètingof, Vizantijskie ikony $v$ Rossii [Les icônes byzantines en Russie], M., 2005. L'histoire de cette icône est dramatique: après la révolution d'Octobre, elle fut transférée au musée de Toropec, d'où elle fut envoyée en 1936 au Musée Russe à Leningrad, où elle fut restaurée. À cause de son très mauvais état, elle ne fut pas intégrée dans l'exposition permanente et n'apparaissait pas même aux expositions temporaires. En 2009, après une demande d'un entrepreneur orthodoxe, l'icône Korsunskaja, malgré toutes les protestations des collaborateurs du Musée Russe, fut transférée à l'église du village d'élite de Sapsan, aux environs de Moscou, où elle se trouve aujourd'hui.

14. Arhimandrit Leonid (Kavelin), « Selo Nikolo-Gostunskoe s ego drevnostjami [Le village de Nikolo-Gostunskoe avec ses antiquités] », Čtenija v Obščestve istorii i drevnostej rossijskih, M., 1861, kn. IV, Smes', p. 187-198.

15. Anna V. Ryndina, «Die "geschnitzte Ikone" im russischen Sakralraum», in Marianne Stößl, éd., Verbotene Bilder: Heiligenfigüren in Russland, München: Hirmer, 2006 (Forschungsheft des Bayrischen Nationalmuseums, 17), p. 110-111.

16. G.N. Melehova «Krestnye hody Kulikova polja (XIX-XX vv.) [Les processions au Champ des bécasses aux XIX ${ }^{\mathrm{e}}-\mathrm{xx} \mathrm{e}^{\mathrm{e}}$ siècles] » http://moseducation.narod.ru/st/melehova/003.htm (dernière consultation le 10 décembre 2011).

17. Melehova, « Krestnye hody Kulikova polja (XIX-XX vv.)».

18. Le chercheur cite une prière pendant l'accouchement, ainsi que le texte du Dit sur l'icône de Nicolas Thaumaturge, de la Martyre Barbara et de Parascève (A.V. Pigin, « Skazanie o ikone Nikolaja Čudotvorca, Varvary Velikomučenicy i Paraskevy Pjatnicy [Le Dit sur l'icône de Nicolas Thaumaturge, de la Martyre Barbara et de Parascève]»), in: Slovar' knižnikov i knižnosti Drevnej Rusi. XVII v. [Dictionnaire des bibliophiles et de la littérature de l'Ancienne Rus'], č. 4. T-Ja. Dopolnenija, SPb., 2004, p. 608.

19. Vladimir, arhimandrit, Opisanie Nikolae-Radovickogo monastyrja [Description du monastère de Saint-Nicolas de Radovicy], 2-e izd. Zarajsk, 1882, p. 49-50.

20. Voir : N.N. Pomerancev, S.I. Maslenicyn, Russkaja derevjannaja skul'ptura [La Sculpture russe sur bois], M., Izobrazitel'noe iskusstvo, 1994; T.M. Kol'cova, Reznye ikonostasy $i$ derevjannaja skul'ptura Russkogo Severa. Katalog vystavki [Les iconostases sculptées et la sculpture en bois de la Russie du Nord, Catalogue de l'exposition], Arhangel'sk, M., 1995; Antonina I. Mordvinova, Derevjannaja cerkovnaja skul'ptura $v$ Čuvašii XVI-načala XXv. Avtoreferat... kandidata iskusstvovedenija [La sculpture religieuse tchouvache en bois, du XVI e jusqu'au commencement du $\mathrm{xx}^{\mathrm{e}}$ siècle), M., 2004; Stößl, éd., Verbotene Bilder. Heiligenfigüren in Russland. 
21. Vladimir, arhimandrit, Opisanie Nikolae-Radovickogo monastyrja, p. 1-47.

22. Ibid., p. 7-8, 57.

23. A.S. Lavrov, Koldovstvo i religija $v$ Rossiii, 1700-1740 [Sorcellerie et religion en Russie, 1700-1740], M., 2000, p. 418, 443.

24. Marianne Stössl, «Hinführung», in Stößl, éd., Verbotene Bilder. Heiligenfigüren in Russland, p. 81, 85. Après la fermeture du monastère de Saint-Nicolas de Radovicy en 1935, l'image se retrouve dans l'église de Sainte-Parascève dans le village de Tugoles (district de Šatura) où elle est vénérée par les croyants. Ainsi, c'est le seul culte parmi ceux étudiés à avoir garder sa popularité jusqu'à maintenant. Poplavskaja, «Palomničestvo, strannopriimničestvo i počitanie svjatyn'», p. 255-260; M. Stössl, «Plastische Heiligenbilder in nachsowjetischer Zeit ", in Stößl, éd., Verbotene Bilder. Heiligenfigüren in Russland, p. 238 ; Mixail Syrčin, «Obraz Svjatitelja i Čudotvorca Nikolaja, imenuemyj Radovickim [L'image du saint thaumaturge Nikolaj, nommé Radovickij]», Žurnal Moskovskoj Patriarhii, 1992, n 7, p. 35.

25. Christine D. Worobec, "Miraculous Healings", in Mark D. Steinberg and Heather J. Coleman, éds., Sacred Stories: Religion and Spirituality in Modern Russia, Bloomington: Indiana University Press, 2007, p. 22-43.

26. Il est intéressant que la collégiale à Epifan', où se trouvait l'icône, soit mentionnée pour la première fois en 1685 (Melehova, «Krestnye hody Kulikova polja»). S'agissait-il aussi d'un culte relativement nouveau?

27. Voir: Andrej E.Petrov, « Èvoljucija pamjati o Kulikovskoj bitve 1380 g. v épohu stanovlenija moskovskogo samoderžavija (rubež XV-XVI vv.): k voprosu o momente transformacii vosprijatija "mesta pamjati" [L'évolution de la mémoire de la bataille du Champ des bécasses en 1380 à l'époque de la formation de l'autocratie moscovite (fin du $\mathrm{XV}^{\mathrm{e}}$-début $\mathrm{du} \mathrm{XVI}^{\mathrm{e}}$ siècle): sur la question du moment de la transformation de la perception du "lieu de mémoire"] », Istoričeskie zapiski, vyp. 7 (125), M., 2004, p. 35-56 ; et aussi : « La mémoire de la bataille de Kulikovo dans l'idéologie de l'État russe des $\mathrm{XV}^{\mathrm{e}}-\mathrm{XVI}^{\mathrm{e}}$ siècles ", Cahiers du Monde russe et soviétique, 46 (1-2), 2005, p. 305-325.

\section{RÉSUMÉS}

Les sources sur lesquelles s'appuie cet article sont relativement peu connues, voire inconnues du milieu scientifique. À ce que nous en savons, les requêtes que soumettaient les gouverneurs pour obtenir l'autorisation de laisser pour un temps les devoirs qui leur avaient été confiés, dans le but d'accomplir un pèlerinage vers le lieu saint de leur choix, n'ont jamais attiré l'attention des chercheurs spécialistes de la religiosité russe. Rédigées selon un formulaire type, ces requêtes sont avares de détails sur les raisons du choix et le caractère du vœu. Cependant, grâce à ces documents, nous disposons presque toujours de données sur le rang du requérant et l'époque du pèlerinage, détail important par rapport au calendrier liturgique. Pour autant, la part étudiée de ces requêtes ne constitue vraisemblablement qu'une fraction de ce que recèlent les fonds de la chancellerie et il semblerait prématuré de tirer des conclusions à ce stade de la recherche. 
The construction of Holy Russia: Muscovite governors' pilgrimages in the seventeenth century This article examines sources that have never attracted scholarly attention, i.e., the petitions of Muscovite governors who asked for permission to leave their duties and go on a pilgrimage to a holy site. These petitions have a strictly formalized structure. They contain laconic data about the reasons governing the choice of shrines and about the nature of the petitioners' vows. They do, however, provide information on the ranks of the petitioners and the times of year when the pilgrimages were to take place. Even the smallest detail is important with regard to the liturgical calendar. However, the petitions studied so far are but a small fraction of the number of petitions held in the archives of the Military Chancellery. It is therefore impossible to make generalized conclusions at this stage of research.

\section{AUTEUR}

\section{ALEKSANDR LAVROV}

Université de Paris IV 